\title{
ORIGINAL RESEARCH \\ Acute Effects of Alcohol on the Human Brain: Diffusion Tensor Imaging Study
}

L.M. Kong

W.B. Zheng

G.P. Lian

H.D. Zhang

\begin{abstract}
BACKGROUND AND PURPOSE: DTI can provide information about brain ultrastructure by quantifying water diffusion. Our objective was to assess the value of DTI in detecting the acute effects of alcohol on healthy human brains.
\end{abstract}

MATERIALS AND METHODS: Sixteen healthy volunteers were studied with conventional MR imaging and DTI before and $0.5,1,2$, and 3 hours after the initiation of acute alcohol administration. Two DTI parameters, FA and ADC, were measured in the frontal lobe, internal capsule, external capsule, precentral gyrus, postcentral gyrus, thalamus, middle cerebellar peduncle, and brain stem. BrACs were measured at each time point after drinking to estimate BACs.

\begin{abstract}
RESULTS: No abnormalities were found by conventional MR imaging at any time point in all subjects. $A D C$ values of the frontal lobe, thalamus, and middle cerebellar peduncle were significantly reduced, reaching a minimum value at 1 or 2 hours, and FA values of the frontal lobe were significantly increased, reaching a maximal value at 0.5 hour in both doses. BrAC (BAC) was significantly increased to reach a peak at 0.5 hour in both doses and decreased gradually.
\end{abstract}

CoNCLUSIONS: DTI can detect changes in brains after acute alcohol consumption that are not detectable by conventional MR imaging. The frontal lobe, thalamus, and middle cerebellar peduncle are more vulnerable to the effects of acute alcohol consumption. DTI is more effective than BrAC or BAC for the detection of alcohol-induced changes on the human brain.
A lcohol abuse mediates its effects on both the developing and developed brain, directly or indirectly, and has acute and chronic complications. ${ }^{1}$ Given the high prevalence of alcohol abuse and the limited and inefficient current treatment options, the need for better understanding of the effects of alcohol is clear. A number of measures have been used to examine these effects. Acute alcohol intoxication has been shown to decrease the amplitude and increase the latency of several components of the ERP, most notably the P3 or P300. ${ }^{2}$ The ERP paradigm was a modified version of the Petrides and Milner Number Sequencing task. ${ }^{3}$ The term "P300" is used to refer to the canonical ERP component, which also is called the P3 or the more nebulous "late positive component." Results from EEG-complexity measures suggest that EEG is a sensitive indicator of task-dependent synchronization characteristics that are modified by low alcohol dose as reflected in alterations in most frequency bands. ${ }^{4}$ Brain imaging studies have shown that acute alcohol administration induces a marked decrease in glucose metabolism throughout the human brain. ${ }^{5}$

Previously MR imaging has been applied to the study of

Received June 25, 2011; accepted after revision July 25

From the Department of Radiology, Second Affiliated Hospital, Medical College of Shantou University, Shantou, China.

This study was supported by the Natural Science Foundation of Guangdong Province, China (grant No. 07008199), and the Science and Technology Planning Project of Guangdong Province, China (grant No. 2010B031600129).

Please address correspondence to W.B. Zheng, MD, Department of Radiology, Second Affiliated Hospital, Medical College of Shantou University, Shantou 515041; e-mail: hwenb@126.com

Indicates open access to non-subscribers at www.ajnr.org

http://dx.doi.org/10.3174/ajnr.A2873 human chronic alcoholism. Early observations by using structural MR imaging studies reported that white matter subjacent to the cortex and the pons is affected in terms of macrostructural volume deficits in uncomplicated alcoholism. Specific brain regions affected by chronic alcohol exposure and depicted by structural MR imaging include the cortical gray and white matter, particularly prefrontal areas in older alcoholic individuals, ${ }^{6}$ and the thalamus. ${ }^{7}$ However, these measures have been unable to explain directly the development of cellular edema after acute alcohol administration.

DTI is a recent application of MR imaging based on measurement of the Brownian motion of water molecules. In DTI, the characteristics of water diffusion in the brain are used to assess the microstructural integrity of white matter pathways; water diffuses more readily along the orientation of axonal fibers than across the fibers due to hindrance from structural elements such as the axolemma and the myelin sheath. ${ }^{8}$ The main parameters measured are FA, which reflects the preferential direction of water diffusion along the white matter tracks, and ADC, which reflects overall diffusivity. DTI has been introduced in the diagnosis of disease conditions such as acute stroke, ${ }^{9}$ multiple sclerosis, ${ }^{10}$ and cerebral ischemia. ${ }^{11}$ In connection with chronic alcohol consumption, many previous DTI studies have reported reduced FA in certain brain regions: for example, in the genu of the corpus callosum; the centrum semiovale ${ }^{12}$; and the frontal and superior sites, such as frontal forceps, internal and external capsules, and fornix. ${ }^{13}$ DTI has revealed evidence for microstructral disruption of white matter in alcoholic men and women, even in regions appearing normal on conventional volume imaging. ${ }^{12,14}$ DTI measures enabled examination of the effect of chronic excessive alcohol consumption on the microstructural integrity of 
major fiber bundles in vivo. To our knowledge, there is no report of DTI to assess the impact of acute alcohol administration on the diffusion of water in the human brain; we hypothesize that DTI can detect the effects of acute alcohol administration in healthy young human brain that are difficult to assess with conventional MR imaging.

\section{Materials and Methods}

Institutional review board approval was obtained for all experiments. All participants provided written informed consent approved by both the university hospital and institutional review boards.

\section{Study Subjects}

Sixteen healthy subjects, 8 men and 8 women, with a mean age of $26 \pm$ 1.1 years, were recruited for the study. We studied the adult volunteers by using a repeated-measures design. The 16 volunteers were randomly divided into 2 groups: a low-dose $(0.45 \mathrm{~g} / \mathrm{kg})$ group $(n=8)$ and a high-dose $(0.65 \mathrm{~g} / \mathrm{kg})$ group $(n=8)$. The alcohol was given in the form of wine (53\% volume of alcohol Maotai wine, 2010, Kweichow Moutai Co. Ltd., Renhuai, Guizhou, China). Subjects in the low-dose group were given $0.45 \mathrm{~g}$ of alcohol per kilogram body weight. That is equivalent to a person of $80-\mathrm{kg}$ weight with 2 glasses of wine $(0.2 \mathrm{~L}, 11 \%$ volume of alcohol). The dose administrated to the high-dose group was $0.65 \mathrm{~g}$ of alcohol per kilogram body weight, which is equivalent to a person of $80 \mathrm{~kg}$ with 3 glasses of wine $(0.2 \mathrm{~L}$, $11 \%$ volume of alcohol). All drinks were mixed with some food, such as peanuts. Each participant received an individually tailored dose of alcohol calculated on the basis of weight following an algorithm: alcohol consumption (milliliters) $=$ the amount of alcohol intake (grams)/alcohol concentration (percentage) $\times 0.8$ (ethanol attenuation). Subjects were asked to consume the wine during a 6- to 10minute period. BrACs were measured before drinking to ensure that participants were not already under the influence of alcohol and immediately before MR imaging to estimate BACs. The Alco-Sensor III breathalyzer (ALCPRO, Knoxville, Tennessee) was used to estimate $\mathrm{BAC}$ and is an electronic instrument that has been found to have good validity and reliability and to provide results highly correlated $(r=$ 0.96 ) with the chemical analysis of blood among cooperative subjects. ${ }^{15}$

Participants were recruited by word of mouth. To be eligible for the study, potential volunteers were interviewed via telephone and asked a number of questions concerning their general health and medical history, in addition to questions especially related to their history of alcohol use and abuse. Potential volunteers with histories of serious physical disease and of psychiatric disorder, seizure, head trauma, or CNS tumors were excluded from participation. To ensure that the alcohol dose received in the study would be within the participants' normal range of experience, we excluded very heavy drinkers. Participants were asked to refrain from any alcohol for 24 hours before their appointment and to eat a light meal 5-6 hours before their appointment. Subjects were informed about the test procedures and consented to participate by signing an informed consent form.

\section{Behavioral Evaluation}

Before alcohol and at $0.5,1,2$, and 3 hours after alcohol administration, subjects were asked to evaluate their subjective sense of headache, excitement, dizziness, sleepiness, or obfuscation.

\section{MR Image Acquisition}

Conventional MR imaging and DTI were performed in all subjects. MR imaging was acquired on a 1.5T MR imaging system (Signa; GE
Healthcare, Milwaukee, Wisconsin) equipped with an 8-channel phased-array head coil. Imaging sequences of the brain included spinecho T1WI, fast spin-echo T2WI, and DTI. The MR imaging parameters were as follows: 2633/23.4 ms (TR/TE) for spin-echo T1WI; 4500/107.9 ms (TR/TE) for fast spin-echo T2WI; and 8000/98.3 ms (TR/TE) and NEX $=1$ for DTI. The other parameters were the following: section thickness $=5 \mathrm{~mm}$ with no gap between sections, matrix $=256 \times 256$, FOV $=24 \times 24 \mathrm{~cm}$. DTI was performed with a single-shot spin-echo echo-planar pulse sequence by using 25 diffusion-encoding directions. The minimum b-value was $0 \mathrm{~s} / \mathrm{mm}^{-2}$; the maximum was $1000 \mathrm{~s} / \mathrm{mm}^{-2}$. Subjects were imaged before alcohol so that each subject could serve as his or her own control. Following the control image acquisition, acute alcohol administration was performed. Volunteers were re-imaged $0.5,1,2$, and 3 hours after the initiation of alcohol administration by using the same imaging parameters as in the prealcohol imaging session. Ear protection was used.

\section{Data Analysis}

Images were postprocessed off-line by using DTIStudio software (Johns Hopkins University, Baltimore, Maryland) and an Advantage workstation for Windows (AW 4.3, GE Healthcare). After correction for movement and EPI-induced distortion artifacts, the diffusion tensor was calculated for each voxel. The final DTI dataset was fed into the software FuncTool, Version 4.5.5 (GE Healthcare), which automatically computes the FA and ADC maps.

The ROIs included the frontal white matter, internal capsule, external capsule, precentral gyrus, postcentral gyrus, thalamus, middle cerebellar peduncle, and brain stem. A series of voxels was selected systematically on the left and right sides of the ROIs in all subjects respectively (Fig 1). Two radiologists who were retrospectively blinded to the follow-up images manually drew the ROIs on each DTI section; the anatomic accuracy of the ROI placement was validated by a board-certified neuroradiologist. The area of each ROI was $32 \mathrm{~cm}^{2}$, except in the external capsule where the ROI areas were $26 \mathrm{~cm}^{2}$. Mean \pm SD of FA and ADC values for the ROIs at $0.5,1,2$, and 3 hours after alcohol administration were recorded.

\section{Statistical Analysis}

All continuous data (FA, ADC value) are expressed as mean $\mathrm{X} \pm \mathrm{SD}$ and were analyzed by repeated-measures analysis of variance followed by individual Student-Neuman-Keuls tests. High- and low-dose effects were compared by using the Student $t$ test. A $P$ value $<.05$ was considered to indicate a statistically significant difference, and data were analyzed by using the Statistical Package for the Social Sciences, Version 15.0 (SPSS, Chicago, Illinois).

\section{Results}

\section{The Effects of Alcohol on the Central Peripheral Nervous System}

A low dose of alcohol changed the mood and behavior of the persons tested. They were in a depressed mood $(n=5)$, had increased speech $(n=4)$, showed signs of excitement $(n=6)$, and had headache $(n=6)$ and dizziness $(n=3)$. The test subjects who had the high dose of alcohol showed reactions like headache $(n=8)$, dizziness $(n=4)$, nausea $(n=4)$, depression $(n=6)$, and obfuscation $(n=2)$. They had problems coordinating and controlling their movements (ataxia).

In our study, peak behavioral effects for both doses of al- 

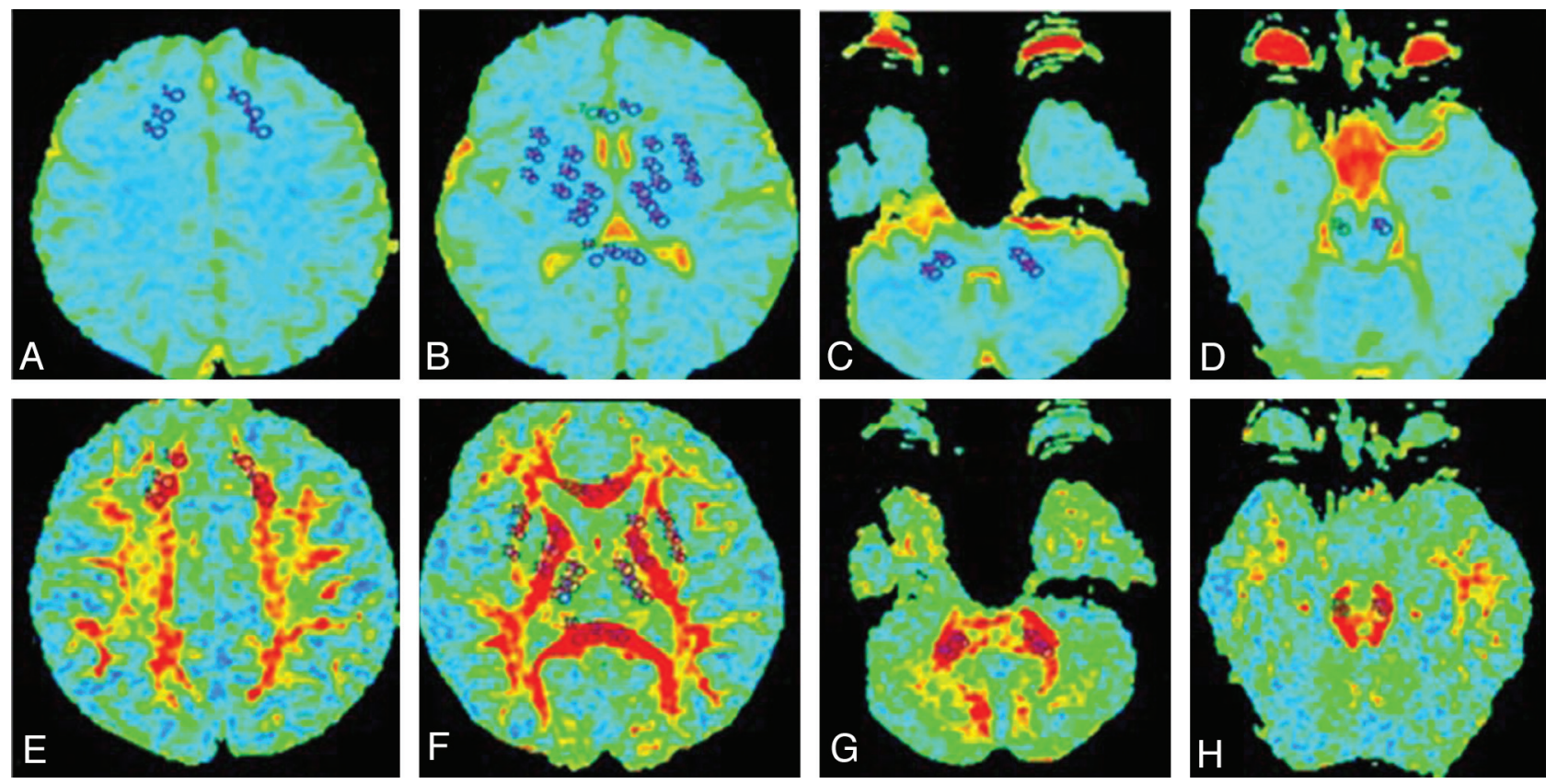

Fig 1. Example of the selection of ROls from 8 structures under study in 1 of the subjects. $A-D, A D C$ maps. $E-H$, FA maps.

cohol occurred around 60 minutes after acute alcohol administration. Both doses increased self-reports for headache, excitement, dizziness, sleepiness, or obfuscation.

\section{MR Imaging}

According to conventional T2WI, no difference in signal intensity between pre- and post-alcohol-consumption subjects was observed, whereas the ADC maps showed regions of hypointensity diffusely scattered throughout in the frontal lobe (Fig 2), thalamus, and middle cerebellar peduncle.

Significant differences were observed for ADC values in the frontal lobe and middle cerebellar peduncle between the prealcohol state and 1 and 2 hours $(P<.01)$ after acute alcohol administration in both the high- and low-dose groups (Tables 1 and 2). The ADC values showed significant changes, reaching a minimum value at 1 hour (high dose: $0.749 \times 10^{-3} \pm$ 0.334; low dose: $\left.0.737 \times 10^{-3} \pm 0.372\right)$ in the frontal lobe followed by a gradual recovery and reaching a minimum value at 2 hours (high dose: $6.414 \times 10^{-3} \pm 0.461$; low dose: $6.527 \times$ $\left.10^{-3} \pm 0.536\right)$ in the middle cerebellar peduncle followed by a gradual recovery in both dose groups. Although the overall effects of both alcohol doses tended to decrease the ADC in the thalamus, reaching a minimum value at 1 hour (high dose: $0.737 \times 10^{-3} \pm 0.309$; low dose: $\left.0.708 \times 10^{-3} \pm 0.254\right)$, the differences were only significant for the high dose $(P<.05)$ (Tables 1 and 2). No statistically significant differences were observed for the ADC values in the other selected regions in both low- and high-dose groups. The dose effect for ADC values was not significant. A sharp decrease of ADC values in the frontal lobes was observed by 0.5 hour after alcohol administration, reaching a minimum value at 1 hour, followed by a gradual recovery in both the low- and high-dose groups (Fig $3 A,-B$ ).

A statistically significant increase in FA was seen in the frontal lobe $(P<.01)$ at 0.5 hour (high dose: $0.595 \pm 0.075$; low dose: $0.588 \pm 0.083)$ after acute alcohol administration compared with before acute alcohol administration in both dose groups. No statistically significant differences were observed for the FA values in the other selected regions in both dose groups (Tables 3 and 4). Figure $3 C,-D$ shows that FA values in the frontal lobes increased and reached a peak at 0.5 hour after drinking. Figure $3 E$ shows the BrACs (BACs) of all 16 subjects; these were significantly increased to a peak at 0.5 hour in both the low-dose, $0.3 \mathrm{mg} / \mathrm{L}(65 \mathrm{mg} / 100 \mathrm{~mL})$, and the high-dose, $0.5 \mathrm{mg} / \mathrm{L}(100 \mathrm{mg} / 100 \mathrm{~mL})$, groups after drinking and then decreased.

Our results showed that the ADC values in the frontal lobe were significantly correlated with the $\mathrm{BrAC}$ at 1 hour $(r=$ $-0.526, P=.030$ ) after acute alcohol administration of a high dose and 3 hours $(r=-0.565, P=.005)$ after a low dose. Our results also showed that the ADC values in the middle cerebellar peduncle were significantly correlated with the $\mathrm{BrAC}$ at 1 hour $(r=-0.681, P=.004)$ after acute alcohol administration of a high dose and the FA values were correlated with the BrAC at 3 hours $(r=0.818, P=.047)$ after acute alcohol administration of a low dose. Table 5 lists the correlation coefficients between the ADC and FA values and the BrAC in the frontal lobe and the middle cerebellar peduncle. There were no significant correlations between the 2 DTI parameters and the BrAC in other ROIs at any time point after the acute alcohol administration.

\section{Discussion}

Ethanol is demonstrated to have many adverse effects on the CNS by depression of hemodynamic and respiratory centers; decrease in regional cerebral blood flow ${ }^{16}$ and resulting cerebral ischemia; formation of free radicals; dysfunction of $\mathrm{Na}^{+}$/ $\mathrm{K}^{+}$-ATPase; and disruption of ionic homeostasis, such as effects on ion influx, including $\mathrm{Na}^{+}, \mathrm{Ca}^{2+}$, and $\mathrm{Mg}^{++} .{ }^{17}$ Because ETOH inhibits $\mathrm{Na}^{+} / \mathrm{K}^{+}$activity, influx of $\mathrm{Na}^{+}$would result in an efflux of $\mathrm{H}^{+}$, with subsequent intracellular $\mathrm{Na}^{+}$ accumulation and eventual cytotoxic edema. ATP levels are 


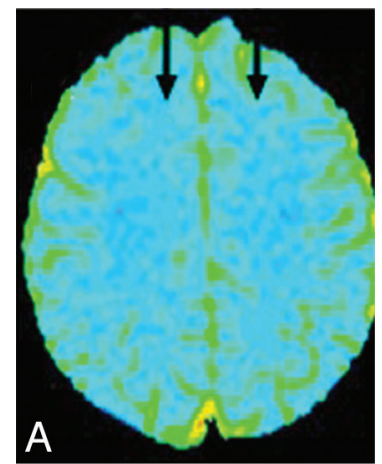

pre-

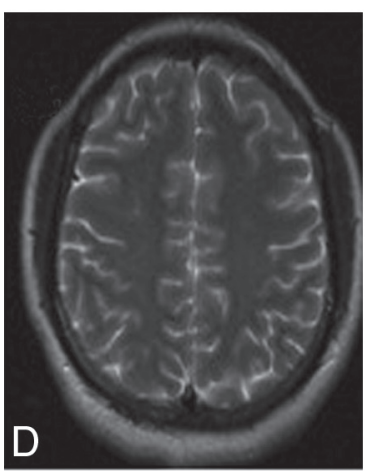

lh-post

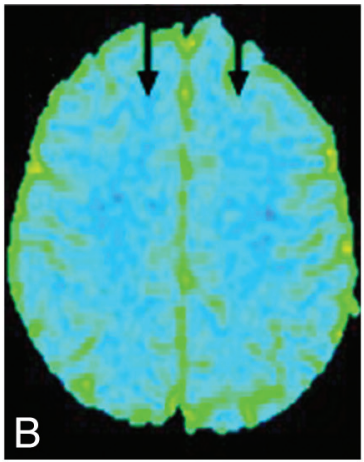

1h-post

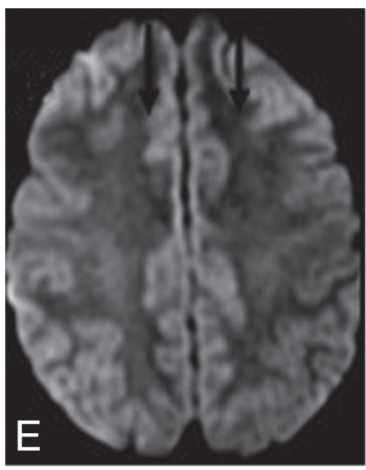

pre-

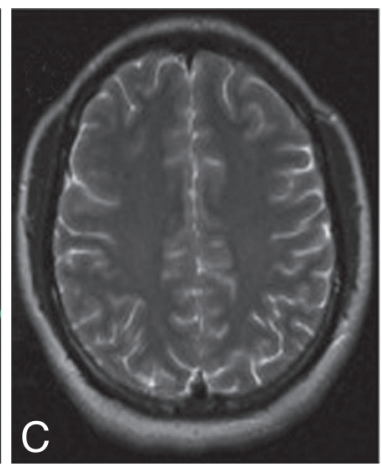

pre-

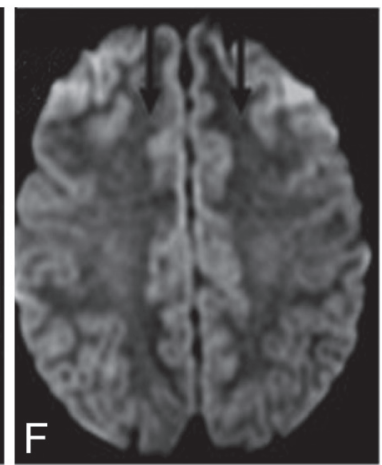

lh-post

Fig 2. $A D C$ maps and T2WI and isotropic DWI images before acute alcohol consumption and 1 hour after the acute alcohol administration in a $25-y e a r-0 l d$ female subject. $A$ and $B$, $A D C$ maps show multiple hypointensities scattered throughout the centrum semiovale at both time points, but there is a marked increase of hypointensity diffusely scattered throughout at 1 hour after acute alcohol consumption compared with before acute alcohol consumption. $C$ and $D, T 2 W I$ images show no visible changes in the centrum semiovale. $E$ and $F$, Isotropic $D$ W images show an increase of hyperintensity diffusely scattered throughout the centrum semiovale at 1 hour after acute alcohol consumption compared with before acute alcohol consumption.

\begin{tabular}{|c|c|c|c|c|c|c|c|c|}
\hline Time & $\begin{array}{c}\text { Frontal White } \\
\text { Matter }\end{array}$ & $\begin{array}{l}\text { Internal } \\
\text { Capsule }\end{array}$ & $\begin{array}{l}\text { External } \\
\text { Capsule }\end{array}$ & $\begin{array}{c}\text { Precentral } \\
\text { Gyrus }\end{array}$ & $\begin{array}{c}\text { Postcentral } \\
\text { Gyrus }\end{array}$ & Thalamus & $\begin{array}{c}\text { Middle Cerebellar } \\
\text { Peduncle }\end{array}$ & Brain Stem \\
\hline Pre- & $0.772 \pm 0.621$ & $0.716 \pm 0.419$ & $0.731 \pm 0.288$ & $0.707 \pm 0.322$ & $0.713 \pm 0.414$ & $0.769 \pm 0.358$ & $0.686 \pm 0.305$ & $0.720 \pm 0.220$ \\
\hline $0.5 \mathrm{hr}$ & $0.753 \pm 0.479$ & $0.721 \pm 0.392$ & $0.747 \pm 0.297$ & $0.699 \pm 0.500$ & $0.715 \pm 0.558$ & $0.764 \pm 0.297$ & $0.674 \pm 0.300$ & $0.718 \pm 0.218$ \\
\hline $1 \mathrm{hr}$ & $0.749 \pm 0.334^{b}$ & $0.714 \pm 0.442^{c}$ & $0.739 \pm 0.346$ & $0.704 \pm 0.359$ & $0.705 \pm 0.420$ & $0.737 \pm 0.309^{b}$ & $0.648 \pm 0.646^{b}$ & $0.704 \pm 0.249$ \\
\hline $2 \mathrm{hr}$ & $0.752 \pm 0.432^{b}$ & $0.722 \pm 0.378^{c}$ & $0.742 \pm 0.293$ & $0.721 \pm 0.589$ & $0.694 \pm 0.349$ & $0.757 \pm 0.351$ & $0.641 \pm 0.461^{b}$ & $0.707 \pm 0.172$ \\
\hline $3 \mathrm{hr}$ & $0.757 \pm 0.471$ & $0.700 \pm 0.394$ & $0.734 \pm 0.381$ & $0.704 \pm 0.405$ & $0.697 \pm 0.292$ & $0.758 \pm 0.206$ & $0.646 \pm 0.427^{b}$ & $0.721 \pm 0.136$ \\
\hline
\end{tabular}

Note:-Pre- indicates Pre-acute alcohol administration.

The numeric values presented are ADC \pm SD for the high-dose group. ADC values are $10^{-3} \mathrm{~mm}^{2} / \mathrm{s}$

${ }^{\mathrm{b}} P<.01$ was considered to indicate an obviously statistically significant difference.

c $P<.05$ was considered to indicate a statistically significant difference.

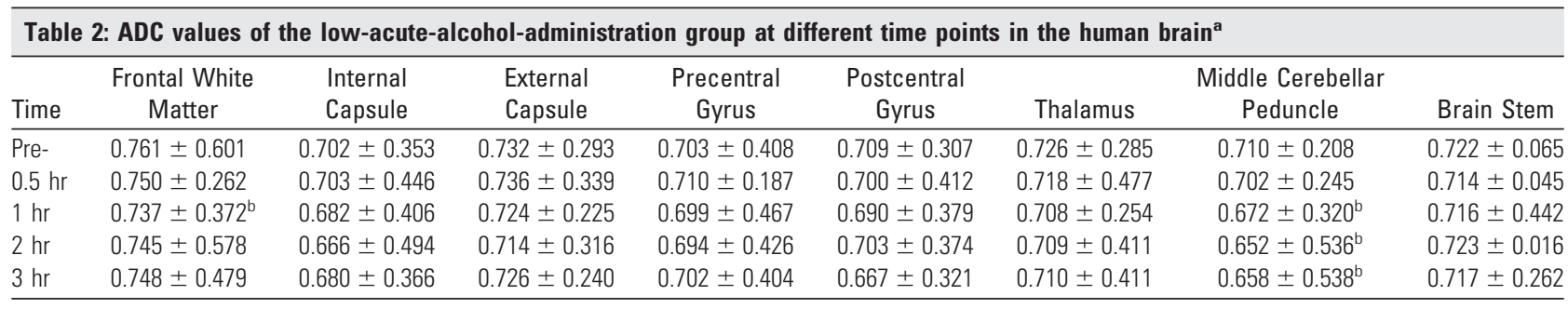

Note:-Pre- indicates Pre-acute alcohol administration.

a The numeric values presented are ADC \pm SD for low-dose group. ADC values: $10^{-3} \mathrm{~mm}^{2} / \mathrm{s}$

b $P<.05$ was considered to indicate a statistically significant difference.

depleted because oxidative phosphorylation is abrogated during ischemia that results from alcohol. Concomitant or progressive energy depletion during ensuing cerebral ischemia propagates $\mathrm{Na}^{+} / \mathrm{K}^{+}$-ATPase failure and aggravates cytotoxic brain edema. In vitro, astrocytes acutely exposed to isosmotic ETOH rapidly swell. Acute exposure to alcohol can induce cellular edema in neonatal rat primary astrocyte cultures and was also associated with a dose-dependent increase in astro- 

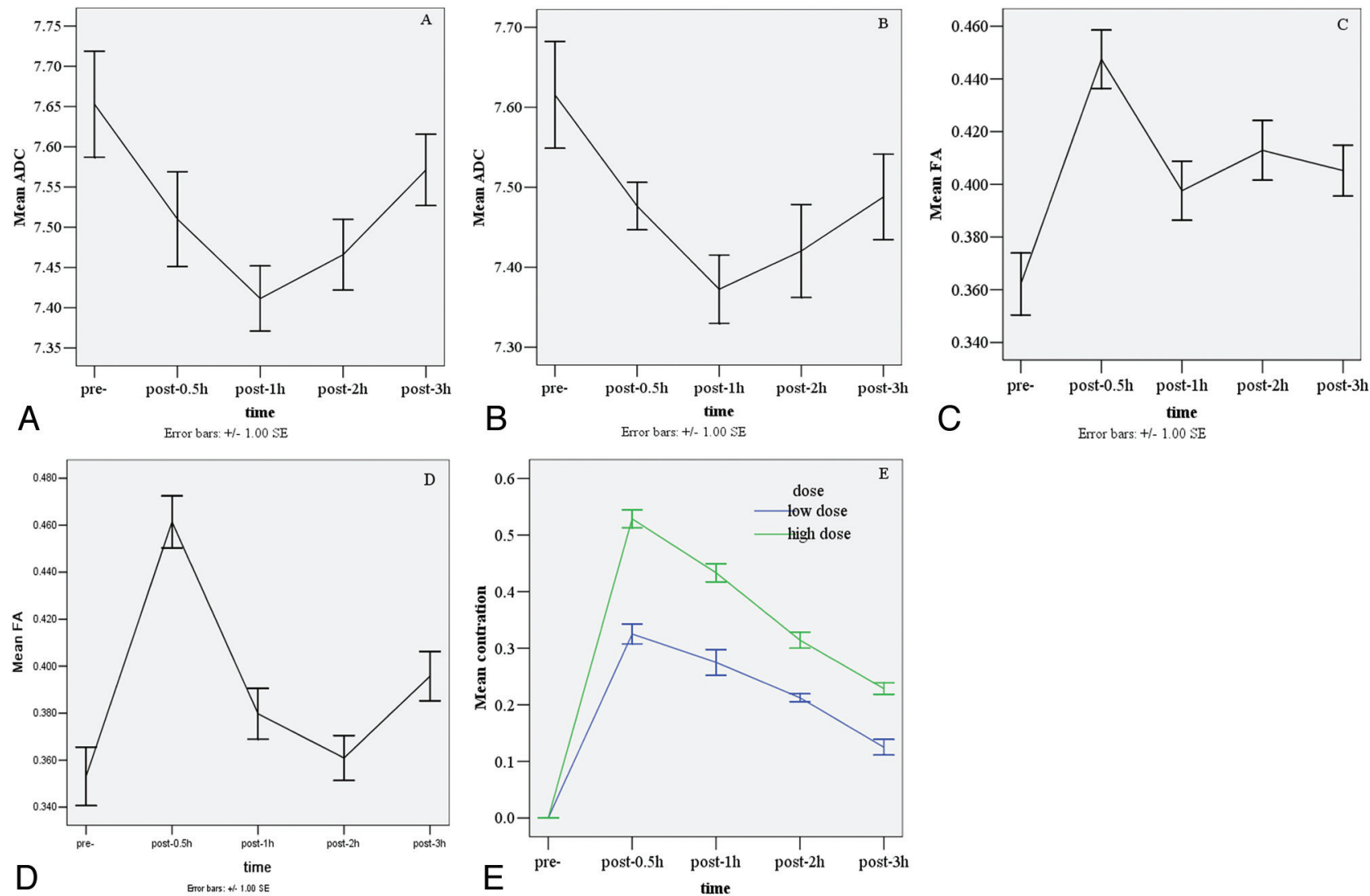

Fig 3. Graphs demonstrate the mean $A D C$ of the high dose $(A)$ and low dose $(B)$, the FA of the high dose $(C)$ and low dose $(D)$ in the frontal lobe, and the $\operatorname{Br} A C$ values $(E)$ for low and high groups in response to 2 doses at different time points.

Table 3: FA values of the high-acute-alcohol-administration groups at different time points in the human brain ${ }^{\mathrm{a}}$

\begin{tabular}{|c|c|c|c|c|c|c|c|c|}
\hline Time & $\begin{array}{c}\text { Frontal White } \\
\text { Matter }\end{array}$ & $\begin{array}{l}\text { Internal } \\
\text { Capsule }\end{array}$ & $\begin{array}{l}\text { External } \\
\text { Capsule }\end{array}$ & $\begin{array}{c}\text { Precentral } \\
\text { Gyrus }\end{array}$ & $\begin{array}{l}\text { Postcentral } \\
\text { Gyrus }\end{array}$ & Thalamus & $\begin{array}{c}\text { Middle Cerebellar } \\
\text { Peduncle }\end{array}$ & Brain Stem \\
\hline Pre- & $0.404 \pm 0.082$ & $0.585 \pm 0.067$ & $0.348 \pm 0.049$ & $0.335 \pm 0.070$ & $0.366 \pm 0.083$ & $0.283 \pm 0.056$ & $0.591 \pm 0.041$ & $0.537 \pm 0.023$ \\
\hline $0.5 \mathrm{hr}$ & $0.445 \pm 0.086^{b}$ & $0.595 \pm 0.075$ & $0.377 \pm 0.053$ & $0.359 \pm 0.075$ & $0.415 \pm 0.097$ & $0.301 \pm 0.048$ & $0.607 \pm 0.046$ & $0.531 \pm 0.029$ \\
\hline $1 \mathrm{hr}$ & $0.438 \pm 0.085^{c}$ & $0.599 \pm 0.072$ & $0.354 \pm 0.059$ & $0.356 \pm 0.070$ & $0.377 \pm 0.088$ & $0.297 \pm 0.050$ & $0.618 \pm 0.064$ & $0.522 \pm 0.035$ \\
\hline $2 \mathrm{hr}$ & $0.444 \pm 0.088^{b}$ & $0.598 \pm 0.076$ & $0.355 \pm 0.059$ & $0.346 \pm 0.097$ & $0.392 \pm 0.069$ & $0.316 \pm 0.039^{c}$ & $0.627 \pm 0.071$ & $0.533 \pm 0.033$ \\
\hline $3 \mathrm{hr}$ & $0.442 \pm 0.079^{b}$ & $0.612 \pm 0.052$ & $0.357 \pm 0.057$ & $0.333 \pm 0.059$ & $0.396 \pm 0.068$ & $0.303 \pm 0.046$ & $0.600 \pm 0.053$ & $0.534 \pm 0.014$ \\
\hline
\end{tabular}

Note:-Pre- indicates Pre-acute alcohol administration.

a The numeric values presented are FA \pm SD for high-dose group.

${ }^{\mathrm{b}} P<.01$ was considered to indicate an obviously statistically significant difference.

${ }^{c} P<.05$ was considered to indicate a statistically significant difference.

Table 4: FA values of the low-acute-alcohol-administration groups at different time points in the human brain ${ }^{\mathrm{a}}$

\begin{tabular}{|c|c|c|c|c|c|c|c|c|}
\hline Time & $\begin{array}{c}\text { Frontal White } \\
\text { Matter }\end{array}$ & $\begin{array}{l}\text { Internal } \\
\text { Capsule }\end{array}$ & $\begin{array}{l}\text { External } \\
\text { Capsule }\end{array}$ & $\begin{array}{l}\text { Precentral } \\
\text { Gyrus }\end{array}$ & $\begin{array}{l}\text { Postcentral } \\
\text { Gyrus }\end{array}$ & Thalamus & $\begin{array}{l}\text { Middle Cerebellar } \\
\text { Peduncle }\end{array}$ & Brain Stem \\
\hline Pre- & $0.353 \pm 0.112$ & $0.534 \pm 0.075$ & $0.338 \pm 0.056$ & $0.360 \pm 0.097$ & $0.384 \pm 0.102$ & $0.280 \pm 0.051$ & $0.585 \pm 0.058$ & $0.542 \pm 0.081$ \\
\hline $0.5 \mathrm{hr}$ & $0.453 \pm 0.090^{b}$ & $0.588 \pm 0.083$ & $0.374 \pm 0.040$ & $0.339 \pm 0.111$ & $0.361 \pm 0.093$ & $0.301 \pm 0.047$ & $0.574 \pm 0.038$ & $0.545 \pm 0.068$ \\
\hline $1 \mathrm{hr}$ & $0.382 \pm 0.095$ & $0.559 \pm 0.096$ & $0.350 \pm 0.059$ & $0.351 \pm 0.088$ & $0.374 \pm 0.080$ & $0.297 \pm 0.056$ & $0.578 \pm 0.054$ & $0.532 \pm 0.094$ \\
\hline $2 \mathrm{hr}$ & $0.358 \pm 0.094$ & $0.524 \pm 0.090$ & $0.351 \pm 0.044$ & $0.352 \pm 0.099$ & $0.379 \pm 0.095$ & $0.282 \pm 0.042$ & $0.619 \pm 0.080$ & $0.523 \pm 0.088$ \\
\hline $3 \mathrm{hr}$ & $0.397 \pm 0.072$ & $0.536 \pm 0.040$ & $0.394 \pm 0.045$ & $0.333 \pm 0.094$ & $0.344 \pm 0.080$ & $0.282 \pm 0.044$ & $0.593 \pm 0.064$ & $0.536 \pm 0.092$ \\
\hline
\end{tabular}

Note:-Pre- indicates Pre-acute alcohol administration

${ }^{a}$ The numeric values presented are FA \pm SD for the low-dose group

${ }^{\mathrm{b}} P<.01$ was considered to indicate an obviously statistically significant difference.

cyte volume. ${ }^{18}$ By definition, brain edema is an abnormal accumulation of fluid within the brain parenchyma that produces a volumetric enlargement of the brain tissue. Currently, 2 major types of brain edema, cytotoxic brain edema and vasogenic brain edema, are recognized. ${ }^{19}$

DTI and ADC could offer the opportunity for real-time detection and differentiation of edema formation. ${ }^{20}$ Although the precise nature (cytotoxic versus vasogenic) of alcohol-dependent edema is still uncertain, here, we evaluated the effects of alcohol on brain Brownian motion of water to assess whether we could document alcohol-induced edema in the human brain by using DTI. 


\begin{tabular}{|c|c|c|c|c|c|c|c|c|}
\hline \multirow{3}{*}{ BrAC } & \multicolumn{4}{|c|}{ High Dose } & \multicolumn{4}{|c|}{ Low Dose } \\
\hline & \multicolumn{2}{|c|}{$A D C$} & \multicolumn{2}{|c|}{ FA } & \multicolumn{2}{|c|}{ ADC } & \multicolumn{2}{|l|}{ FA } \\
\hline & $r$ & $P$ & $r$ & $P$ & $r$ & $P$ & $r$ & $P$ \\
\hline 1-hr FL & -0.526 & $.030^{\mathrm{b}}$ & -0.089 & .642 & 0.148 & .436 & -0.081 & .671 \\
\hline 1-hr МСР & -0.681 & $.004^{\mathrm{b}}$ & 0.316 & .234 & -0.277 & .506 & -0.438 & .278 \\
\hline 2-hr FL & 0.142 & .396 & -0.129 & .447 & 0.018 & .889 & 0.077 & .548 \\
\hline 2-hr MCP & -0.256 & .338 & 0.253 & .344 & 0.698 & .054 & 0.364 & .376 \\
\hline 3-hr FL & 0.216 & .216 & 0.199 & -.129 & -0.565 & $.005^{b}$ & -0.098 & .655 \\
\hline 3-hr MCP & 0.108 & .690 & 0.224 & .405 & -0.746 & .077 & $0.818^{b}$ & .047 \\
\hline
\end{tabular}

Note:-FL indicates frontal lobe; MCP, middle cerebellar peduncle.

a $P<.05$ indicates a statistically significantly correlation between the measurable ADC and $\mathrm{FA}$ values and the BrAC

${ }^{b}$ Correlation is significant at the .05 level (2-tailed).

ADC should be interpreted in terms of changes in the diffusion coefficient of the extracellular space and in its fractional volume relative to the intracellular volume. ADC values were helpful for identifying and distinguishing the type of edema in ischemia. ${ }^{21}$ Reduced ADC suggests cytotoxic edema, whereas increased ADC in the brain indicates vasogenic edema. ${ }^{22}$ The ADC decrease seen in abnormal brain tissue can be ascribed to cellular swelling (cytotoxic edema), in which water shifts from the extracellular to the intracellular compartment because of disturbances in ion homeostasis.

The most important result of our study is that ADC values in the frontal lobe, thalamus, and middle cerebellar peduncle were predictive of the development of cytotoxic edema. Reduced ADC values with both alcohol doses in the frontal lobe and middle cerebellar peduncle and also high alcohol dose in the thalamus after drinking suggest development of cytotoxic edema, whereas increased ADC may suggest gradual recovery of edema and may reach normal 3 hours after acute alcohol consumption in the frontal lobe and thalamus.

FA, a normalized measure of anisotropy calculated from the diffusion tensor, has been shown to be sensitive to microstructural changes in white matter integrity. ${ }^{23}$ The anisotropic diffusion is thought to increase as a result of myelination, axonal thickness, or the amount of parallel organization of axons or a combination of these 3 factors. In our study, the FA values were not significantly changed; alterations were found only in the frontal lobe. The frontal lobe showed increased mean FA values in 6 subjects and decreased mean value in 2 subjects with the high dose, and an increased mean FA value in 6 subjects, a decreased mean value in 1 subject, and no alteration in 1 subject with the low dose. However, no statistically significant alteration in FA values was observed in other selected ROIs.

FA values in our experiment appeared to increase temporarily in the frontal lobe in both the low- and high-dose groups. The time dependence of the FA value did not exhibit the same monotonic trend (Fig $3 C,-D$ ). Our finding may be explained by the fact that the acute increase in FA value was thought to arise from cell swelling during cytotoxic edema, which, for white matter, reduced the spacing between the myelin fiber bundles. The increased extracellular tortuosity may cause increased restriction of water movement transverse to the fiber direction and thus an increase in the FA value. ${ }^{24}$ Therefore, the FA value of the ROIs may be more influenced by the location of the cerebral lesion than its extent and severity, which should be investigated in the future with a large number of patients. In white matter tracts, the direction of the largest eigenvalue represents diffusivity along the longitudinal axis of the tract (axial diffusivity, $\lambda 1$ ), which can be altered with the disruption of axonal integrity and axonal deletion; the 2 smaller eigenvalues ( $\lambda 2$ and $\lambda 3$ ) represent the radial diffusivity, which increases selectively with a decline in myelin integrity. ${ }^{25,26}$ The calculation of ADC and FA is derived by algebraic manipulation of the eigenvalues. However, analysis of the impact of alcohol on the component eigenvalues of the diffusion tensor received no attention in our study. To enhance the use of indices of anisotropy as reliable indicators of the severity of acute alcohol administration in clinical practice, a more detailed study of the intrinsic values of these parameters will be required.

The fact that alterations of ADC and FA values showed in the frontal lobes, thalamus, and middle cerebellar peduncle in our study supports the concept that these areas are more vulnerable to the effects of acute alcohol consumption. Frontal lobes compose the single largest cortical region in the brain. The frontal lobe is involved in functions such as decisionmaking; creative thinking; artistic expression; aspects of emotional behavior; and spatial working memory, language, and motor control. ${ }^{27}$ The thalamus serves as a major junctional complex that modulates input to the prefrontal cortex, and it has been suggested that the prefrontal cortex should be defined on the basis of its anatomic relationship with the medial dorsal thalamic nucleus. ${ }^{28}$ The ventrointermediate nuclei is the thalamic relay of the cerebellothalamocortical tract; it sends projections primarily to the motor cortex. This pathway is important for movement control. ${ }^{29}$ The middle cerebellar peduncle is a fiber tract originating in the pontine nuclei, which send axons to the contralateral cerebellar cortex; most of these axons pass through the contralateral middle cerebellar peduncle. This intimately connected pathway presumably sends somatosensory movement-related information to the cerebellum. ${ }^{30}$ Decreased activation of the cerebellum under acute alcohol conditions has previously been reported ${ }^{30}$ and may underlie impairments seen in mood, behavior, cognition, and motor activity. ${ }^{32}$

The average peaks of the BrAC in both dose groups, which roughly occurred approximately 0.5 hour after the onset of drinking, represent the peak of blood-ethanol concentrations. Therefore, in our study, how the brain is influenced by acute alcohol administration may not be reflected by BrAC or BAC; DTI is more effective than BrAC or BAC for the detection of alcohol-induced changes in human brain.

\section{Conclusions}

Our investigation demonstrates that DTI can provide detailed information concerning brain Brownian motion of water in cases of acute alcohol consumption, allowing the diagnosis of cytotoxic brain edema. DTI is capable of detecting changes in the brain after acute alcohol consumption not visible on conventional MR imaging. DTI has been shown to be more effective in detecting alcohol-induced changes on the human brain compared with BAC or BrAC. The frontal lobes, thalamus, and middle cerebellar peduncle are especially vulnerable to the effects of alcohol. The recovery of DTI parameters (ADC and 
FA values) after drinking may indicate that both low and high doses of alcohol can affect the function of the brain temporarily but not damage it irreversibly for those who are not chronic alcoholics or heavy drinkers.

\section{References}

1. Alderazi Y, Brett F. Alcohol and the nervous system. Current Diagnostic Pathology 2007;13:203-09

2. George MR, Potts G, Kothman D, et al. Frontal deficits in alcoholism: an ERP study. Brain Cogn 2004;54:245-47

3. Petrides M, Milner B. Deficits on subject-ordered tasks after frontal- and temporal-lobe lesions in man. Neuropsychologia 1982;20:249-62.

4. Molnár M, Boha R, Czigler B, et al. The acute effect of low-dose alcohol on working memory during mental arithmetic. II. Changes of nonlinear and linear EEG-complexity in the theta band, heart rate and electrodermal activity. Int J Psychophysiol 2009;73:138-42

5. Volkow ND, Wang GJ, Franceschi D, et al. Low doses of alcohol substantially decrease glucose metabolism in the human brain. Neuroimage 2006;29: 295-301

6. Cardenas VA, Studholme C, Gazdzinski S, et al. Deformation-based morphometry of brain changes in alcohol dependence and abstinence. Neuroimage 2007;34:879-87

7. Bellis D, Narasimhan A, Thatcher DL, et al. Prefrontal cortex, thalamus, and cerebellar volumes in adolescents and young adults with adolescent-onset alcohol use disorders and comorbidmental disorders. Alcohol Clin Exp Res 2005;29:1590-600

8. Niogi SN, Mukherjee P, McCandliss BD, et al. Extent of microstructural white matter injury in postconcussive syndrome correlates with impaired cognitive reaction time: a $3 \mathrm{~T}$ diffusion tensor imaging study of mild traumatic brain injury. AJNR Am J Neuroradiol 2008;29:967-73

9. Fiebach JB, Jansen O, Sartor K, et al. Serial analysis of the apparent diffusion coefficient time course in human stroke. Neuroradiology 2002;44:294-98

10. Filippi M, Cercignani M, Comi G, et al. Diffusion tensor magnetic resonance imaging in multiple sclerosis. Neurology 2001;56:304-11

11. Lythgoe MF, Busza AL, Calamante F, et al. Effects of diffusion anisotropy on lesion delineation in a rat model of cerebral ischemia. Magn Reson Med 1997;38:662-68

12. Rosenbloom M, Sullivan EV, Pfefferbaum A. Using magnetic resonance imaging and diffusion tensor imaging to assess brain damage in alcoholics. Alcohol Res Health 2003;272:146-52

13. Pfefferbaum A, Rosenbloom M, Rohlfing T, et al. Degradation of association and projection white matter systems in alcoholism detected with quantitative fiber tracking. Biol Psychiatry 2009;65:680-90. Epub 2008 Dec 21

14. Yeh PH, Simpson K, Durazzo TC, et al. Tract-based spatial statistics (TBSS) of diffusion tensor imaging data in alcohol dependence: abnormalities of the motivational neurocircuitry. Psychiatry Res 2009;173:22-30. Epub 2009 May 12
15. Borges $\mathrm{G}$, Cherpitel C, Mittleman M. Risk of injury after alcohol consumption: a case-crossover study in the emergency department. Soc Sci Med 2004;58: 1191-200

16. McDonough KH, Giaimo ME, Gentilello LM, et al. Low-dose ethanol alters the cardiovascular, metabolic and respiratory compensation for severe blood loss. J Trauma 2002;53:541-48

17. Yamakami I, Vink R, McIntosh TK, et al. Effects of acute ethanol intoxication on experimental brain injury in the rat: neurobehavioral and phosphorus-31 nuclear magnetic resonance spectroscopy studies. J Nerosurg 1995;82:813-21

18. Aschner M, Mutkus L, Allen JW. Aspartate and glutamate transport in acutely and chronically ethanol exposed neonatal rat primary astrocyte cultures. Neurotoxicology 2001;22:601-05

19. Cernak I, Vink R, Zapple DN. The pathobiology of moderate diffuse traumatic brain injury as identified using a new experimental model of injury in rats. Neurobiol Dis 2004;17:29-43

20. Hanstock CC, Faden AI, Bendall MR, et al. Diffusion-weighted imaging differentiates ischemic tissue from traumatized tissue. Stroke 1994;25:843-48

21. Schlaug G, Siewert B, Warach S, et al. Time course of the apparent diffusion coefficient (ADC) abnormality in human stroke. Neurology 1997;49:113-19

22. Cernak I, Vink R, Zapple DN, et al. The pathologies of moderate diffuse traumatic brain injury as identified using a new experimental model of injury in rats. Neurobiol Dis 2004;17:29-43.

23. Mukherjee P, McKinstry RC. Diffusion tensor imaging and tractography of human brain development. Neuroimaging Clin N Am 2006;16:19-43

24. Sotak $\mathrm{CH}$. The role of diffusion tensor imaging in the evaluation of ischemic brain injury: a review. NMR Biomed. 2002;15:561-69

25. Sun SW, Liang HF, Le TQ, et al. Differential sensitivity of in vivo and ex vivo diffusion tensor imaging to evolving optic nerve injury in mice with retinal ischemia. Neuroimage 2006;32:1195-204

26. Newcombe VF, Williams GB, Menon DK, et al. Analysis of acute traumatic axonal injury using diffusion tensor imaging. Br J Neurosurg 2007; 21:340-48

27. Semendeferi K, Damasio H, Frank R, et al. The evolution of the frontal lobes: a volumetric analysis based on three-dimensional reconstructions of magnetic resonance scans of human and ape brains. J Hum Evol 1997; 32:375-88

28. Moselhy HF, Georgiou G., Kahn A. Frontal lobe changes in alcoholism: a review of the literature. Alcohol Alcohol 2001;36:357-68

29. Yamada K, Akazawa K, Nishimura T, et al. MR imaging of ventral thalamic nuclei. AJNR Am J Neuroradiol 2010;31:732-35

30. Kim J, Lee SK, Lee JD, et al. Decreased fractional anisotropy of middle cerebellar peduncle in crossed cerebellar diaschisis: diffusion-tensor imagingpositron-emission tomography correlation study. AJNR Am J Neuroradiol 2005;26:2224-28

31. Gundersen H, Gruner R, Hugdahl K, et al. The effects of alcohol intoxication on neuronal activation at different levels of cognitive load. Open Neuroimag J 2008;2:65-72. Epub 2008 Aug 22

32. Volkow ND, Ma Y, Wang GJ, et al. Moderate doses of alcohol disrupt the functional organization of the human brain. Psychiatry Res 2008;162:205-13 